\title{
Linx
}

Revue des linguistes de l'université Paris X Nanterre

5 | 1994

La négation

\section{La négation : de l'absence au refus, et du refus à l'absence}

Laurent Danon-Boileau

\section{(2) OpenEdition}

Journals

Édition électronique

URL : http://journals.openedition.org/linx/1205

DOI : 10.4000/linx.1205

ISSN : 2118-9692

Éditeur

Presses universitaires de Paris Nanterre

\section{Édition imprimée}

Date de publication : 1 juin 1994

Pagination : 177-189

ISSN : 0246-8743

\section{Référence électronique}

Laurent Danon-Boileau, «La négation : de l'absence au refus, et du refus à l'absence », Linx [En ligne], 5 | 1994, mis en ligne le 18 juillet 2012, consulté le 01 mai 2019. URL : http://journals.openedition.org/ linx/1205; DOl : 10.4000/linx.1205

Ce document a été généré automatiquement le 1 mai 2019.

Département de Sciences du langage, Université Paris Ouest 


\title{
La négation: de l'absence au refus, et du refus à l'absence
}

\author{
Laurent Danon-Boileau
}

1 L'objet du présent article est de proposer une analyse de la négation fondée en partie sur l'observation du langage de l'enfant. Chemin faisant, bien entendu, je mettrai en écho des considérations plus générales.

2 Je poserai, sans chercher à l'argumenter, l'existence de deux opérations fondamentales de négation : d'une part le constat d'absence ( «Il n'y a pas de pain dans la huche »); celui-ci a d'emblée partie liée avec l'aspect. Et de l'autre le refus ( « Non je ne veux pas»), lequel implique la mise en jeu de la représentation de l'autre, de l'intersubjectivité, et donc, en termes linguistiques, de la modalité. J'analyserai chacun des deux types de négation pour eux-mêmes, puis je chercherai à préciser l'articulation qu'il est possible d'en donner.

\section{La négation d'absence}

3 Lorsqu'il apparaît dans le langage de l'enfant, un marqueur comme « apu », constitue tout ensemble une préfiguration de l'aspect et de la négation d'absence. Je vais ici développer la dimension qui touche plus directement à la négation d'absence.

4 La négation d'absence ( «il n'y a pas de pain dans la huche») est une opération de jugement de nature assertive, au même titre que l'affirmation correspondante « il y a du pain dans la huche». On ajoute en général que l'affirmation est première, et que la négation repose sur une affirmation préconstruite. En somme dire « il n'y a pas de pain dans la huche » est une opération en deux temps

- premier temps : je préconstruis l'existence du pain dans la huche

- second temps : je nie la validité de ce préconstruit.

5 Cette façon de dire a l'avantage de préserver l'intuition que toute négation procède d'une affirmation préalable, mais elle a l'inconvénient de ne pas suffisamment distinguer le statut modal de l'affirmation préconstruite de celui de la négation qui lui fait suite. 
6 Pour éviter de faire de la négation la superposition de deux jugements de même nature, on peut évidemment poser qu'affirmation et négation partagent un même contenu de pensée, un même dictum, qui n'est en lui-même ni affirmé ni nié, puis que ce contenu est associé soit à un modus affirmatif (et l'on est conduit à l'affirmation) soit à un modus (et l'on est conduit à un énoncé négatif). Cette seconde façon de voir laisse toutefois échapper l'intuition d'un préconstruit positif formant la base du jugement de négation.

7 Ce qui fait problème dans l'une et l'autre interprétation, c'est en fait la nature du préconstruit qui sert de base au jugement explicite de négation : on ne peut le concevoir ni comme un contenu de pensée asserté positivement, ni comme un contenu de pensée dépourvu de tout modus (c'est-à-dire suspendu ou neutre). Pour lui donner son plein statut il me semble nécessaire d'avoir recours à la notion d'anticipation. Cette notion dérive directement de l'article de Freud sur la Dénégation. Pour Freud la négation est l'expression d'un écart entre ce que l'on anticipait (ce à quoi l'on s'attendait, ce que l'on souhaitait, ce que l'on désirait) et ce que l'on constate : «Ce dont je croyais qu'il était là parce que je le désire n'est pas là à l'instant présent ». Dans cette interprétation, la négation se fonde sur une positivité préalable, mais cette positivité n'est pas un jugement de réalité, elle est une anticipation sur l'état de la situation d'énonciation : "il n'y a pas de pain dans la huche » veut dire « je m'attendais à ce qu'il y ait du pain dans la huche [je le désirais], et je constate qu'il n'y en a pas ». Envisagée ainsi, la modalité du préconstruit sous-jacent à la négation est une modalité de type déontique ( « je veux, je m'attends à ce que, je souhaite qu'il y ait du pain dans la huche ») et non pas une modalité aléthique ou ontique. Et ce que traduit la négation c'est la discordance entre cette anticipation préconstruite sur le mode déontique et la réalité constatée au moment de l'énonciation. Cette discordance, elle, est donnée sur le mode aléthique ou ontique car il s'agit d'un jugement de fait. Il n'y a donc pas, au fondement de la négation une assertion. Il n'y a pas non plus un contenu de pensée qui ne serait ni positif ni négatif et que l'on orienterait ensuite soit vers le positif (assertion) soit vers le négatif (négation). Il y a une anticipation d'un état de la réalité. La modalité correspondant à cette anticipation peut être identifiée à une modalité non aléthique. Et dans son ensemble la négation d'absence apparaît alors comme l'articulation de deux modus de nature radicalement différents :

- un premier modus optatif (déontique, non aléthique) qui permet la préconstruction correspondant à « je m'attendais à, je souhaitais que... »

- un second modus aléthique cette fois qui marque l'écart entre la représentation préconstruite et la situation d'énonciation.

8 Dans son ensemble, la négation d'absence permet à l'énonciateur de fournir une interprétation de la réalité en disant qu'elle équivaut pour lui à l'absence de ce à quoi il s'attendait : ici comme dans bien d'autres cas il s'appuie sur ce qui se voit (la situation au moment de l'énonciation) pour faire partager à l'autre ce qui ne se voit pas (la représentation correspondant à son attente).

9 On peut reprendre dans cette perspective l'analyse des marqueurs de la négation « ne » et « pas » en français. A propos de « ne » Damourette et Pichon parlent de discordance, dans la pensée de l'énonciateur. Ce marqueur, disent-ils, a même effet que le «ne » de "je crains que Paul ne vienne ». Dans ce dernier exemple, «ne " marque un conflit dans la pensée que je forme relativement à la venue de Paul: d'un côté j'anticipe cette venue mais de l'autre je la redoute. De même, dans un énoncé négatif tel que «Paul n'est pas là ", le «ne" conserve cette valeur de discordance et souligne l'écart entre mon anticipation de la présence de Paul, et le constat que je fais de son absence. 
10 Reste l'interprétation du «pas ». Dans les propositions de Damourette et Pichon, ce marqueur est décrit comme un "forclusif » c'est-à-dire comme une façon de construire un contenu de pensée en soulignant son caractère d'inactualité, de non-conformité à la situation présente, d'altérité ou d'extériorité par rapport à celle-ci. On sait que "pas » appartient à une série de termes de quantité au nombre desquels on compte également " point » " goutte ", « mie ", " miette ». Avant de devenir traces d'opérations, ces mots désignent chacun une quantité infime de quelque chose. A ce titre ils ont une valeur complexe : une miette de pain, par exemple, est tout ensemble une quantité positive de pain, mais c'est également trop peu de pain. Associés à «ne» la valeur de ces termes se simplifie. Ils perdent leur valeur de quantité minimale, et marquent simplement l'absence d'une quantité suffisante, manière indirecte de renvoyer au souhait qui la voudrait plus abondante. Ainsi dans «ne... pas», "pas» assied le modus d'une représentation d'anticipation, tandis que "ne " souligne l'écart institué entre cette anticipation et la situation effective du moment de l'énonciation.

\section{Négation de refus}

11 A côté de la négation d'absence, il existe un second type de négation qui correspond au refus. C'est celui auquel Spitz donnait pour ancêtre le mouvement céphalogyre négatif du nourrisson. Dans le développement de son expression chez l'enfant, on peut distinguer plusieurs étapes. A un premier stade, celui des tout débuts du langage, quand il est encore difficile de distinguer entre énoncé et expression accompagnant le geste, entre énoncé et poursuite langagière de la mimique, la négation intervient dans des situations très particulières : celles dans lesquelles l'enfant se plie à regret à une interdiction parentale. Ainsi un enfant, en s'écartant d'un radiateur brûlant, pourra accompagner son mouvement de retrait d'un hochement de tête et d'un «Non! » distinct, répétant ainsi l'interdiction qui lui a été formulée. La première négation de refus n'est donc pas la marque d'une simple opposition à la volonté des parents.

Plusieurs problèmes se posent concernant le statut de l'énoncé qui correspond à ce type de refus. Est-ce un simple écho de l'interdit parental ? Sans doute pas, ou alors c'est un écho plein de sens, un écho enrichi. Tout se passe comme si l'enfant tentait de se persuader du bien-fondé de l'interdiction, et cherchait à se détourner lui-même de son projet initial de toucher le radiateur, projet qui pourtant continue à l'attirer. Son " Non » n'est donc ni un écho sonore à la parole de la mère, ni une simple conduite d'imitation mais une tentative, par le recours à l'imitation de la conduite verbale, pour intérioriser la parole d'un autre, et obéir à l'interdit en s'identifiant à celui qui l'énonce : il y a imitation si l'on veut mais imitation confuse : l'enfant «joue » le discours de sa mère et joue la confusion imaginaire avec elle ; mais en se pliant à l'injonction, il sait aussi qu'il n'est pas elle, et le marque explicitement en lui obéissant. En tout état de cause, le premier " non » de l'enfant n'est pas un «non » d'opposition, mais une façon de s'identifier à celle qui dit non, afin de surmonter un conflit de désir entre ce que l'enfant souhaite de lui-même et ce qu'il se sent contraint de souhaiter pour conserver l'amour de son objet d'amour.

13 Au bout du compte, comment modéliser le support de la négation formulée par l'enfant? Il s'agit d'un acte de citation au discours direct dans lequel celui qui cite ne se distancie pas explicitement de celle qu'il cite, et l'on peut même penser que la confusion est mise au profit pour résoudre le conflit. Le sujet symbolique que constitue l'enfant ne parvient pas encore à se dégager de celle à laquelle il s'adresse. Et s'il est malaisé de configurer la 
relation entre celui qui parle et celui auquel il s'adresse, une chose est sûre c'est qu'ils ne sont pas extérieurs l'un à l'autre. La négation de refus ne part pas d'une situation dans laquelle s'affronteraient deux sujets nettement distincts.

On pourrait penser que cette façon de dire "non ", cette façon de parler est une pratique de la toute petite enfance, et un phénomène qui n'intéresse pas le discours de l'adulte.

Je ne le pense pas. Nous nous serons tous surpris, une fois à l'autre, à répéter une question qui nous était adressée pour nous donner le temps de la comprendre et de l'intérioriser, avant d'y répondre. Quel statut accorder alors à ces énoncés préalables à la réponse ? Dans un échange tel que :

Pierre : Est-ce qu'il faut remettre la clef au concierge ?

Louis Remettre la clef au concierge, je ne sais pas. Je ne crois pas, on ne m'a rien dit.

Quand Louis dit « Remettre la clef au concierge? ? il y a pour une part une demande de confirmation, mais je ne pense pas qu'un tel énoncé puisse être seulement entendu comme un «Est-ce que j'ai bien entendu? Tu me demandes si l'on doit remettre la clef au concierge... » Je ne pense pas que ce soit une question-écho. Répéter « remettre la clef au concierge » comme le fait Louis me semble plutôt une façon de dire «j'avoue que je ne m'étais pas posé la question, laisse-moi réfléchir, laisse moi faire mienne cette question que tu me poses ». On constate au passage que cette façon de parler permet de construire un segment de discours sur un mode très particulier puisqu'il n'est en somme ni affirmé ni nié et ni assumé par l'énonciateur, ni rejeté à l'extérieur de lui : c'est en fait la construction du thème dont il s'agit.

17 Mais revenons au discours de l'enfant. Après le stade du «non » que je viens de gloser, on trouve la mise en place de la franche négation de refus. Reprenant l'exemple de toute à l'heure, il faudrait supposer que l'enfant qui dit «non" persiste cette fois dans son intention première de toucher le radiateur. Un «non » de cet ordre suppose que l'enfant soit assez assuré de l'amour de sa mère (et de ses propres représentations) pour pouvoir tenter de lui déplaire.

Cela ne veut pas dire pour autant que l'expression du refus dans son ensemble se soit parfaitement unifiée. S'opposant à l'ordre qui lui est fait d'aller au lit, quand un enfant dit «dodo non » (par exemple) tout se passe comme si «dodo» constituait encore dans la bouche de l'enfant une citation des propos de la mère, une reprise de son désir à elle, pour n'être qu'ensuite associée à l'expression de son propre refus : le refus de l'enfant ne trouve à s'exprimer qu'en s'adossant à une parole qui lui est énonciativement étrangère.

Il y a toutefois début d'organisation entre les deux: d'une part un lien anaphorique s'établit, au sein de l'énoncé de l'enfant, entre le « non » et le « dodo », puisque ce « non » porte sur « dodo ». D'autre part on conçoit que même si « dodo » demeure une citation au discours direct de la mère, cette parole n'est plus immédiatement superposable à celle qui exprimait l'ordre initial. le fait que l'enfant s'oppose à l'ordre qu'il reprend vient troubler la valeur injonctive attachée à «dodo». «Dodo» exprime alors une représentation « conflictuelle » qui renvoie tout à la fois de l'enjeu du débat ( «aller au lit» ou « ne pas aller au lit»). En quoi l'enfant n'est pas loin de s'aviser que ceux qui disputent ne remettent pas en cause la représentation de ce dont ils disputent, mais que leur différend porte seulement sur les qualités que chacun assigne au thème.

20 Au terme du mouvement, "Dodo » est alors doublement suspendu : à soi seul, sans le «non » dont il est assorti, il n'exprime plus le souhait de l'une ou l'autre partie, non plus qu'un quelconque état de la réalité. Il vaut pour un contenu de représentation indiquant 
seulement ce dont parlent ensemble mère et enfant. Dans le registre de la négation d'absence, la progression symbolique se marque si le mot perd son pouvoir magique de garantir le retour de la chose. Dans le cas du refus, il faut que le mot se détache du désir de l'un ou l'autre pour signifier l'enjeu du débat commun.

Progressivement le statut de l'hétérogénéité du discours évolue car cette hétérogénéité parvient à se représenter. Et je souhaiterais caractériser ce mouvement.

L'hétérogénéité d'emploi de "Dodo non ", on l'a dit, rappelle le statut d'un énoncé qui insère une citation au discours direct. Dans l'absolu rien ne permet de distinguer une citation directe d'un énoncé qui n'est pas rapporté. Ce sont les conditions d'emploi qui varient entre l'un et l'autre, et le fait que l'énonciateur cesse tout à coup de parler en son nom pour parler au nom d'un autre. Mais cette variation ne fait l'objet d'aucune inscription formelle dans l'énoncé. Au discours indirect, en revanche, le contenu des propos rapportés (au niveau shifters, temps, modes, par exemple), fait l'objet de variations qui soulignent la représentation de l'hétérogénéité, et son intégration dans les paroles de l'énonciateur primaire.

La syntactisation complète de l'hétérogénéité correspondant à l'énoncé de refus, celle qui correspondrait au discours indirect, me parait acquise quand le refus de l'enfant s'exprime non plus par un «Dodo non » mais par un «Pas dodo ». Ce qui la signe, c'est tout autant le passage du «non» au «pas» que le changement de place de l'élément négatif. Dans «pas dodo » il devient possible à l'enfant de commencer par un élément (" pas ») qui d'emblée annonce le conflit d'intérêt et de jugement, en sorte que « dodo » n'est plus alors ni un ordre ni un refus mais l'expression pleine d'une «notion ». Même si dans « Pas dodo », " dodo » est reprise d'un mot réellement prononcé par la mère, il n'y plus citation de son acte énonciatif. Dire "pas dodo ", ce n'est plus bâtir son refus sur l'énonciation explicite de l'autre, mais construire une modalité négative (pas) sur un contenu prédicatif (dodo) qui n'est plus, en soi, pourvu d'aucune modalité. Dans les faits, très souvent, lorsque «pas dodo " s'est définitivement substitué à « dodo non » on voit surgir des formules comme: «dodo, veux pas », lesquelles permettent au sujet de se poser explicitement comme support d'une modalité radicale ("veux pas »). A ce point, le sujet peut poser son discours comme lieu d'articulation de projets en conflits : le projet de sa mère de l'envoyer au lit, et le sien propre de rester éveillé. En sorte que dans un énoncé tel que « dodo, veux pas » « dodo » est pleinement un thème. Il renvoie à une notion déjà en circulation dans l'interlocution. Cette notion ne fait l'objet d'aucun jugement, l'enfant ne pose plus « dodo" ni comme intention explicite de la mère, ni non plus, bien sûr, comme une intention à lui. La question de savoir si dodo veut dire « dodo-oui » ou « dodonon ", demeure en suspens. Il y a " épochè » au sens husserlien. On pourrait le gloser par : "Question d'aller au lit, pas question! ». Cette mise en suspens qui caractérise le thème se situe dans un double registre :

1 - d'une part il y a suspens au niveau de l'opposition affirma-tion/négation ou existence/ non existence. On en trouve la marque dans le langage adulte à plus d'un titre :

- en termes d'existence/suspension d'existence si le segment apposé est de type nominal («Question poisson, j'adore la sole » versus «*Question du poisson, j'adore la sole » «*La question du poisson, j'adore la sole », ou encore « Comme poisson, j'adore la sole »/ * «Comme un poisson j'adore la sole »)

- ou en terme de validation/suspension de validation si le segment apposé est de type verbal ( Qu'il soit en retard, ça ne m’étonne pas »/ «*Qu'il est en retard, ça ne m’étonne pas », « Etre en retard, c'est son habitude »/ "*D'être en retard, c'est son habitude ».). 
A cet égard, le thème est entre le oui et le non, ente l'existence et la non-existence. Dans le dialogue, si le thème est reprise, il n'est pas pour autant reprise d'une construction référentielle (anaphore) ou modale (assertion) préalable.

2 - il y a par ailleurs suspension au niveau du support énonciatif de ce thème. Ce n'est ni vraiment «toi» ni vraiment «moi» qui parle. On est dans le registre d'une intériorisation de la dyade locuteur/colocuteur. Ce que Culioli désigne par la dyade énonciateur/coénonciateur.

Je voudrais ici souligner deux caractéristiques de la négation de refus qui l'opposent assez profondément à la négation d'absence : d'une part contrairement à ce qui se passe avec la négation d'absence, où il s'agit pour le sujet de prendre la mesure de l'inactualité - c'està-dire du statut « représentatif » de sa pensée - ici, ce qui est mis en jeu par le sujet qui dit "non» est d'emblée pour lui une représentation de ce que l'autre pense, d'un actualisable, non d'une actualité. Dire non c'est s'opposer à ce que l'autre entend vous faire faire mais qui n'est pas encore. D'autre part, la représentation qui sert de point de départ au refus n'émane pas du sujet lui-même. « Non » est une réponse à une proposition que l'on décline et qui n'émane pas de soi. En sorte que pour dire «non» il faut que l'enfant dispose de représentations suffisam-ment stables pour tolérer tant l'absence d'actualisation que la conflictualité des valeurs. Et qu'en outre il n'ait plus à s'étayer comme sujet symbolisant dans le regard de sa mère puisque cette fois il s'agit d'exprimer une divergence.

\section{La négation polémique}

La négation dont il vient d'être rendu compte me semble assez proche de ce que l'on trouve dans le discours de l'adulte avec la négation polémique.

Si l'on considère un énoncé tel que « Pour moi, Jean n'est pas un acteur » on s'avise que le rejet qu'elle contient ne peut avoir de sens que pour autant que celui qui s'exprime s'accorde à reconnaître que Jean, s'il n'a pas les mérites qui feraient de lui un acteur véritable, en a du moins la fonction. Le paradoxe réside en ceci qu'il faut créditer Jean de ce dont précisément on entend le priver. Il ne viendrait à l'idée de personne de dire d'un metteur en scène célèbre, Fellini par exemple, « Pour moi, Fellini n'est pas un acteur ». En revanche dans ce cas une exclamation telle que " pour moi, Fellini n'est pas un metteur en scène » est parfaitement admissible.

L'expression du refus, loin d'être une opération simple par laquelle l'énonciateur repousse ce qu'on lui propose, signe l'émergence progressive d'un conflit entre deux mouvements : celui qui conduit, au nom de la coénonciation assumée, à endosser un contenu de pensée véhiculé dans l'échange, et celui par lequel l'énonciateur se dégage de cette adhésion. La négation signe la dualité du sujet de l'énonciation. Il ne s'agit pas tant d'un écart entre deux contenus de représentations que d'un écart « dans » l'énonciateur entre lui-même en tant qu'il valide et qu'il ne valide pas l'attribution d'un prédicat $\mathbf{p}$ à un sujet $\mathbf{s}$, selon qu'il coénonce et/ou qu'il énonce. D'où le fait que le support de la négation de "refus » ne peut pas s'envisager comme une entité circonscrite opposée à un " autre " qui lui serait extérieur. Le support modal d'une négation de refus conjugue consensualité (sur la nature de l'objet jugé) et divergence de la part de l'énonciateur par rapport à cette adhésion préconstruite. Le sujet modal ici construit se démarque de la consensualité mais prend appui sur elle. 
29 Ainsi, dans la négation de refus de l'enfant comme dans la négation polémique, le sujet d'énonciation ne constitue pas un support modal circonscrit opposé à un autre. Il est tout à la fois support de la coénonciation (lui et l'autre) et de l'écart qu'il construit par rapport à cette coénonciation.

\section{Articulation des deux sources de la négation}

On vient de prendre successivement en compte les deux types de négation que constituent l'absence et l'expression du refus. Il se trouve que la plupart du temps (et en tout cas en français) ce sont les mêmes marqueurs qui permettent la construction de l'un et de l'autre. D'où vient que l'expression de l'absence et celle du refus se croisent pour finir sur le symbole de la négation ? D'où vient que « apu » et «non » finissent par se dire par «ne... pas»? Dans ce qui va suivre, mon propos sera de saisir comment opère l'articulation entre l'un et l'autre. Je procéderai pour ce faire par un retour au langage de l'enfance, en me fondant sur le journal d'acquisition tenu par Mireille Brigaudiot qui figure en annexe à sa thèse.

31 Un jour son fils Guillaume prend dans une main le mouchoir qui lui sert d'objet transitionnel, et dans l'autre un mouchoir qui lui ressemble. Puis il se caresse la joue alternativement avec l'un puis avec l'autre en disant pour l'un «Doudou», et « Doudou non » pour l'autre.

En termes psychanalytiques on pourrait dire que cet enfant est parvenu à un stade où il a assez investi l'autre de ce qu'il aime ou de ce qui l'intéresse pour en pouvoir constater la présence. Dire « Doudou non » c'est constater la présence de ce qui n'est pas un doudou. On est je crois dans une illustration de ce qui pourrait être la validation du «presque Doudou ». L'enfant signifie tout à la fois qu'il reconnait la proximité et la différence du second mouchoir avec son objet transitionnel. Mais si l'on suit les formes qu'emprunte le langage, on est amené à penser que ce nouvel état de la problématique de la négation correspond à une introjection de l'état de la coénonciation à l'œuvre dans l'expression du refus. Voici comment : pour employer le terme de « doudou » afin de désigner/qualifier le mouchoir qui n'est pas son doudou, il faut bien que l'enfant accepte que la représentation dont il use réponde en quelque manière au contenu de sens du lexème " doudou ", lors même que ses propos veulent écarter ce mauvais mouchoir de la sphère de ce qui est «doudou ». En cela, tout se passe comme si l'enfant opérait à part soi une «négation polémique ", laquelle le conduisait à repérer le mauvais mouchoir (celui qui n'est pas son objet transitionnel) sur le mode d'une coénonciation fictive, puisqu'il se démarquait de cette coénonciation en disant «non ». Cette interprétation de " Doudou non » équivaut à formuler les choses comme si l'enfant disait "Certes nous pourrions appeler cela un doudou, mais pour ma part je m'écarte de cette façon de voir les choses ». Ou bien, quelques années plus tard « Vous allez me dire qu'il s'agit de mon doudou, mais ce n'est pas mon doudou». C'est en ceci je crois qu'il y a introjection de la polémique. L'introjection réside en ce que la négation ne marque plus un écart de la subjectivité cherchant à se constituer en marge de la coénonciation, mais au contraire une feinte de la subjectivité mimant cette rupture pour penser une propriété négative. Car dans ce premier temps de l'expression de la différence entre les mouchoirs, pour que la qualité de « non-doudou » ait droit de cité, il faut que la "doudouité » ait été fictivement rapportée au support modal que constitue la coénonciation, tandis que l'énonciateur rétablit préten-dûment la vérité des choses en s'écriant « doudou, non » à l'endroit du mouchoir 
menteur. Il semblerait donc que pour accéder à la construction de cette représentation conflictualisée de la qualité d'un objet (quelque chose qui est et qui n'est pas « doudou ») l'enfant doive préserver la dimension positive de la représentation en la plaçant dans la coénonciation, sinon en l'autre qui est en lui : c'est à ce prix qu'il se ménage de convoquer par le nom de doudou quelque chose dont il souhaite souligner qu'en vérité ce ne l'est pas.

Ce détour permet de revenir à la relation qu'un sujet définit entre un mot et la chose qui peut lui correspondre. Cette relation ne fonctionne pas en tout ou rien. A côté d'énoncés tels que «Ceci est une pipe " (l'intérieur de la notion correspondant à " pipe ») ou « Ceci n'est pas une pipe » (l'extérieur de la notion) il existe des énoncés tels que « Ceci n'est pas vraiment une pipe " (frontière de la notion) ou bien encore "Ceci est vraiment une pipe " (centre attracteur de la notion). Tout ceci laisse présager l'existence d'un espace notionnel qui part de ce qui n'est absolument pas une pipe pour venir sur ce qui est presque une pipe, ou une pipe à la rigueur, et finir sur ce qui est une "pipe par excellence ». Ce sont là des problèmes que Culioli a maintes fois abordés. Si je les évoque, c'est pour souligner qu'à mon sentiment la diversification qu'il introduit dans la structure de la notion ne s'explique pas en termes de contenu. De ce point de vue, elle ne peut pas se représenter sur un mode topologique, spatialisé, comme une figure fermée définissant un intérieur un extérieur et une frontière, car les oppositions relevées ont fondamentalement trait à la forme que revêt le jugement ainsi qu'à la nature de son support modal. Ainsi l'extérieur d'une notion, ce qui est du côté du " pas vraiment mais presque, un peu si tu veux, mais pas tout à fait pour moi » ne peut pas être différencié de son intérieur en termes de contenu de représentation: il n'existe rien qui « objectivement » soit presque un acteur sans l'être vraiment. Ce dont il s'agit et que ces gloses désignent c'est la place que l'énonciateur se construit comme support du jugement qu'il énonce.

\section{Propriété négative, thème et prédication}

Le journal de Guillaume mentionne un autre développement de ce glissement du refus vers l'expression d'une qualité négative. Feuillant un livre, l'enfant désigne du doigt une page de texte en disant «ti ( =ici/ça) écrit », puis un dessin qui figure en regard en disant «ti non écrit». Plutôt qu'une qualité de l'une puis de l'autre page, ce qu'expriment ces énoncés jumelés, c'est une comparaison, laquelle est tout à la fois différence et identité. Le mouvement permet en effet de construire une référence stable, celle du thème commun aux deux énoncés, la «page de livre» que «ti » désigne en chacun; puis il permet ensuite de faire varier la propriété distinctive associée à chacune (être écrit ou pas). Il s'ensuit alors, dans chaque énoncé une différence de statut entre thème et prédicat : celui-là est stable et constant, celui-ci est divers, en sorte que dans «ti non écrit » l'existence de ce que désigne « ti » se trouve soustrait à l'empire de la négation qui affecte le prédicat « écrit ».

On entrevoit ici ce qui conduit l'enfant à recourir, pour la caractérisation d'un objet, pour exprimer qu'un objet ne dispose pas d'une qualité, à la négation en «non " plutôt qu'à celle en "pas » : pour lui "pas X » appartient au système de la négation d'absence et absorbe immanquablement $X$ dans le vide : à ses yeux « pas gâteau » signifie «il n'y a pas de gâteau, il n'y a rien » et ne saurait encore signifier « ce n'est pas du gâteau, c'est de la biscotte ». A ses yeux, seule la négation issue du refus permet de préserver l'existence 
d'un thème pour lui associer en un second temps un prédicat négatif. « Non » indique une divergence d'appréciation sur la chose jugée mais la laisse subsister. "pas » la ferait disparaître.

Bien entendu, dire "non écrit» au lieu de "pas écrit " constitue une réduction de l'éventail des qualités d'une page d'un livre à la seule alternative d'être écrite ou de ne l'être pas. Rien n'est encore distingué dans l'ordre du «non-écrit ». Cela ne veut pas dire que l'enfant ne soit pas en mesure de percevoir la différence entre une page blanche et un dessin par exemple. Mais que, lorsqu'il se centre sur la représentation de la page écrite, la distinction - du côté de ce qui n'est pas écrit - entre ce qui est page blanche et ce qui est dessin n'opère pas encore. "Pas» en revanche ouvrirait l'éventail de nouvelles classifications du côté de la négation.

Comment en vient-on alors à l'expression de la négation tel qu'elle figure dans un énoncé banal du type "Le bébé ne dort pas »? Certes, il s'agit d'un état de langage beaucoup plus tardif. Il suppose cette fois que l'enfant soit en mesure d'articuler explicitement et dans le même registre un jugement d'existence positif (le jugement d'existence relatif au sujet, le bébé) et un jugement d'attribution négatif (le fait que ce bébé ne dort pas). Il s'agit cette fois de penser de la même façon l'absence d'une chose, et l'absence, en une chose, d'une propriété qu'on lui voudrait assigner.

38 Certains enfants ont de grandes difficultés à parvenir à l'expression de ce dernier type de négation. Un jeune garçon jouant avec des figurines représentant des animaux, simule une bataille entre un éléphant et un lion. L'éléphant tue le lion. Puis, dans un revirement, l'enfant décrète la résurrection du lion, "mort non ", me dit-il en désignant le lion, comme si tout à coup c'était moi qui avait souhaité le tuer tandis qu'il en assurait la survie. le «non " de «mort, non» contrairement au "pas» de "pas mort» permet à l'enfant de simuler le refus d'une idée qui ne viendrait pas de lui. Une petite fille constatant que contrairement à son thérapeute elle ne porte pas des chaussures qui se lacent (lesquelles sont pour elles des «baskets ») pourra dire de manière analogue " moi basket non" en un énoncé à mi-chemin entre le constat et de la reprise d'une interdiction, car « moi basket non » c'est tout autant « moi les chaussures que je porte ne se lacent pas » que «moi, on n'a pas voulu que je porte des chaussures à lacets comme les grands parce que je ne sais pas les nouer, et ma mère m'a dit "basket, non", tes chaussures doivent êtres des «non-baskets » des chaussures qui ne se lacent pas ». Cette glose un peu longue sans doute fait ressortir que cet énoncé est plutôt du côté de la prédication négative ( mes chaussures sont des non-baskets») que du côté de la suspension de prédication ( $«$ mes chaussures ne sont pas des baskets mais d'autres sortes de chaussures»). Pour qu'il y ait véritablement suspension de la prédication, dénonciation du lien entre le sujet et le prédicat, il faut plusieurs choses : d'une part que l'existence du sujet soit conçue comme indépendante de la validation du lien prédicatif. D'autre part que la validation de ce lien puisse être pensé sur le même mode que l'existence ou la non-existence d'un objet. C'est alors seulement que l'on passe de l'attribution à une chose d'une propriété négative au constat d'absence en cette chose d'une propriété positive. A ce point, l'on est sorti de la dimension argumentative. Il s'est produit un « désintéressement de la négation " parallèle à celui qui permettait de passer tantôt de « encore » à « apu ». C'est ce désintéressement, au demeurant permet d'ouvrir un nouvel espace symbolique.

On le voit, pour que la négation parvienne à s'établir sous la forme qu'on lui connait, il faut qu'un certain nombre de « stades » symboliques aient pu être franchis. Il faut d'abord 
que la question de l'existence de l'objet qui supporte de la propriété puisse être posée indépendamment de l'existence en l'objet de propriété elle-même. Cela se fait, semble-til, si l'enfant parvient à contraster deux objets de nature proche dont l'un dispose de la propriété en cause mais non l'autre. La comparaison tout à la fois renforce la permanence de l'objet et l'indépendance de la propriété. Dans un premier temps, quand la négation porte sur la propriété, il ne s'agit pas d'un constat d'absence en l'objet de la propriété, mais comme un refus de décerner cette propriété à l'objet (lequel est fiction de polémique). Puis le refus évolue en véritable prédication de propriété négative.

Pour qu'advienne enfin le constat en l'objet de l'absence de la propriété il faut encore un mouvement qui permette de délaisser toute dimension altercative, et rapporte l'absence de propriété au format de l'absence d'objet. Mais alors, ce qui permet de statuer sur l'absence n'est plus la situation d'énonciation; c'est en fait le thème, objet second, déjà construit par le discours. Dire l'absence d'une propriété en un objet n'est pas constater une absence dans la situation d'énonciation, c'est déjà opérer une construction au second degré, indépendante, en un sens, de ce qui est ou n'est pas à l'instant ou se tient le discours. La construction du thème permet cette émancipation. Il découle également de là la possibilité de comparer les choses entre elles pour définir des propriétés différentes et non simplement « opposables».

\section{BIBLIOGRAPHIE}

Brigaudiot, M., 1990, Acquisition du langage, les premiers mots, Thèse Paris VII.

Culioli, A., 1990, Pour une théorie des opérations énonciatives, t.1, Ophrys, Gap.

Freud, S., 1925, « Die Verneinung », GW, XIV,15.

\section{RÉSUMÉS}

L'objet du présent article est de montrer le développement des marques de négation chez l'enfant (entre deux et trois ans). Il rapporte la négation à deux sources le constat d'absence d'une part, le refus de l'autre. Il montre que la notion de refus est une notion complexe dont le maniement suppose de la part de l'enfant une capacité à se distinguer de son interlocuteur. La négation nexale au sens de Jespersen ('Ceci n'est pas mon ours') est proposée comme le résultat de l'articulation des deux sources initialement définies pour l'opération, plus précisément comme la dérive d'un refus fictif en constat d'absence partiel.

The aim of the article is to follow the development of negation in the language of the child. It tends to show that there are two sources namely the statement of absence and that of refusal. It underlines the fact that refusal is quite a complex operation which implies a capacity to differentiate oneself from one's co-speaker. It also tends to consider nexal negation, as defined by Jespersen, (e.g. "That is not my bear") as the development of a simulated refusal into a partial statement of absence. 
AUTEUR

LAURENT DANON-BOILEAU

Paris III et Centre Alfred Binet 